PAPER

\title{
Applicability and relevance of models that predict short term outcome after intracerebral haemorrhage
}

\author{
M J Ariesen, A Algra, H B van der Worp, G J E Rinkel
}

J Neurol Neurosurg Psychiatry 2005;76:839-844. doi: 10.1136/jnnp.2004.048223

See end of article for authors' affiliations

.....................

Correspondence to:

Dr A Algra, Department of

Neurology and Julius

Centre for Health Sciences

and Primary Care,

University Medical Centre

Utrecht, Str. 06.131, PO

Box 85500,3508 GA

Utrecht, the Netherlands;

A.Algra@umcutrecht.nl;

http://www.juliuscenter.nl

Received 22 June 2004

Revised version received

6 September 2004

Accepted

20 September 2004
Objectives: Several models for prediction of short term outcome after intracerebral haemorrhage (ICH) have been published, however, these are rarely used in clinical practice for treatment decisions. This study was conducted to identify current models for prediction of short term outcome after ICH and to evaluate their clinical applicability and relevance in treatment decisions.

Methods: MEDLINE was searched from 1966 to June 2003 and studies were included if they met predefined criteria. Regression coefficients of multivariate models were extracted. Two neurologists independently evaluated the models for applicability in clinical practice. To assess clinical relevance and accuracy of each model, in a validation series of 122 patients the proportion with a $\geqslant 95 \%$ probability of death or poor outcome and the actual 30 day case fatality in these patients were calculated. Receiver operator characteristic (ROC) curves were computed for assessment of discriminatory power.

Results: A total of 18 prognostic models were identified, of which 14 appeared easy to apply. In the validation series, the proportion of patients with $a \geqslant 95 \%$ probability of death or poor outcome ranged from $0 \%$ to $43 \%$ (median 23\%). The 30 day case fatality in these patients ranged from $75 \%$ to $100 \%$ (median 93\%). The area under the ROC curves ranged from 0.81 to 0.90 .

Conclusions: Most models are easy to apply and can generate a high probability of death or poor outcome. However, only a small proportion of patients have such a high probability, and 30 day case fatality is not always correctly predicted. Therefore, current models have limited relevance in triage, but can be used to estimate the chances of survival of individual patients. ntracerebral haemorrhage $(\mathrm{ICH})$ represents about $12 \%$ of all strokes. ${ }^{1}$ Short term prognosis of patients with spontaneous ICH is poor, and about $50 \%$ of patients who experience an ICH die within 30 days. ${ }^{2-4}$ Early survival of patients with ICH, in general, is known to be strongly dependent on the Glasgow Coma Scale (GCS) score on admission. Other factors that are known to predict outcome after ICH are size of the haemorrhage and presence of intraventricular haemorrhage. ${ }^{5}$

Prediction of outcome in patients with ICH can be used for two main purposes:

(1) in an emergency department to differentiate between patients who might still benefit from intensive care and those who have such a poor prognosis that they will not benefit from intensive care any more. Thus, in this sense, outcome prediction can be used for making decisions about starting intensive treatment or not.

(2) to inform patients and relatives about the chances of recovery.

Several models have been developed for prediction of short term outcome after ICH, but, to our knowledge, these are rarely used for triage in clinical practice. Additionally, previous authors have noted that no grading scale for ICH is consistently used for triage and acute intervention in either clinical care or clinical research. ${ }^{6}$

The aims of the present study were:

- to identify existing models for the prediction of short term outcome after primary ICH

- to evaluate whether these models can be rapidly and easily applied at the time of presentation

- to evaluate whether the prediction by the models is valid and accurate enough to base major treatment decisions upon for a relevant proportion of all admitted patients
- to assess the discriminatory power of the models in the estimation of a patient's prognosis.

This allowed us to evaluate the correctness of the estimate of a patient's prognosis over the whole range of outcome probabilities.

\section{METHODS}

\section{Literature search}

We searched MEDLINE from 1966 to June 2003 for studies in which a prognostic model was described for short term outcome after ICH. The following search strategy was used: "Cerebral haemorrhage" [Medical Subject Headings $(\mathrm{MESH})]$ AND "Prognosis" [MESH] NOT ("Animals" [MESH] OR "Animal" [MESH] OR "Models, Animal" [MESH] OR "Infant" [MESH] OR "Infant, Newborn" [MESH] OR "Craniocerebral Trauma" [MESH] OR "Injuries" [Subheading] OR "Cerebrovascular Trauma" [MESH] OR "Head Injuries, closed" [MESH] OR "Brain Injuries" [MESH]). Bibliographies of retrieved articles were examined for further relevant publications. This method of cross-checking was continued until no further publications were found.

\section{Inclusion criteria}

Studies were included if:

- the described multivariate model was developed in patients with primary ICH

- short term poor outcome was defined as death or dependence measured within six months after ICH. Dependence was defined as a score of 3-5 on the modified Rankin scale ${ }^{78}$ or a score of 2 or 3 on the GCS ${ }^{9}$

Abbreviations: GCS, Glasgow Coma Scale; ICH, intracerebral haemorrhage 
- studies presented a logistic regression model with corresponding intercept and regression coefficients. If the intercept was not reported, the regression coefficients, the probability of outcome, and data on predictors had to be reported to allow calculation of the intercept

- the publications were in English, French, German, or Spanish.

We focused on predictive models that were applied soon after the first clinical and radiological assessment. Therefore, those studies in which surgery or change of a predictor over time was included in the model were excluded. Furthermore, we excluded studies of patients with ICH as result of thrombolysis, trauma, or operation.

\section{Data extraction}

The following information was extracted from each study: number of patients, definitions of predictors, definition of outcome, time of outcome assessment, regression coefficients of the prognostic model, and the intercept. If the intercept was not reported, it was calculated as follows: Intercept $=-\mathrm{LN}((1 / \mathrm{p})-1)-\left(\beta_{1}{ }^{*}\right.$ predictor $_{1}+\ldots \beta_{\mathrm{n}}{ }^{*}$ predictor $\left._{\mathrm{n}}\right)$, where $p$ is the overall proportion of outcome in the study population and predictor $_{1}$ to predictor $_{n}$ are the means of these predictors. This formula is derived from the logistic regression equation.

\section{Validation series}

We applied the prognostic models to data of 122 patients aged 18 years and older who had been admitted to our hospital with an ICH between January 1988 and December 1997. Patients were included if:

- they had been admitted with a primary ICH (supratentorial only) within 72 hours after the onset of symptoms

- the score on the GCS on admission could be retrieved from the records

- a computed tomography (CT) scan of the brain had been performed in our hospital immediately after admission.

During the study period, 306 patients were admitted to our hospital with an ICH. A total of 184 patients were excluded for the following reasons: insufficient CT scan data $(n=173)$ (CT scan performed in another hospital $(n=122)$, performed

\begin{tabular}{|c|c|}
\hline \multicolumn{2}{|c|}{$\begin{array}{l}\text { Electronic search: } \\
1096 \text { studies }\end{array}$} \\
\hline \multicolumn{2}{|c|}{$\begin{array}{c}\text { Title screening: } \\
127 \text { studies possibly relevant } \\
969 \text { studies excluded } \\
\text { (complications of ischaemic infarctions, surgical trials, case reports) }\end{array}$} \\
\hline \multicolumn{2}{|c|}{$\begin{array}{c}\text { Detailed evaluation abstract: } \\
39 \text { studies probably relevant } \\
88 \text { studies excluded } \\
\text { (long term outcome, language, thrombolysis related) }\end{array}$} \\
\hline $\begin{array}{r}\text { Detailed evaluation full paper (cros } \\
18 \text { studies incl } \\
24 \text { studies } \\
\text { (no multivariate } \\
\text { no logistic regressio } \\
\text { surgery inclu }\end{array}$ & $\begin{array}{l}\text { s-checking references in } 3 \text { studies) } \\
\text { uded (table } 1) \\
\text { excluded } \\
\text { model }(n=11) \text {, } \\
\text { n/intercept }(n=9) \\
\text { ded }(n=4))\end{array}$ \\
\hline
\end{tabular}

Figure 1 Method of selection of studies. outside time window $(n=30)$, incomplete scans $(n=16)$, or missing CT scan $(n=5))$ and GCS not retrievable $(n=11)$.

For each patient, we retrieved the following data from the medical records: sex, age, systolic and diastolic blood pressures, and the GCS score at admission. From the CT scan at admission we calculated the ICH volume, intraventricular spread, presence of intraventricular haemorrhage, and the septum pellucidum shift. Hydrocephalus was considered as present when the bicaudate index exceeded the upper limit of normal per decile of age. ${ }^{10}$

To evaluate the discriminatory performance of the models in the estimation of a patient's prognosis, we computed a receiver operating characteristic (ROC) curve for each model and assessed its area under the curve (AUC) (SPSS for Windows, Standard version released 15 November 2001). ${ }^{11}$ In an ROC curve the true positive proportion (sensitivity) is plotted against the false positive proportion ( 1 - specificity). With ROC curves the prediction of outcome over the whole range of outcome probabilities (from $0 \%$ to $100 \%$ ) can be evaluated. The area represents the probability that a randomly chosen diseased subject is correctly rated or ranked with greater suspicion than a randomly chosen non-diseased subject. ${ }^{12}$ An AUC of 1 corresponds with a perfect prediction and an AUC of 0.5 with no discriminatory power at all.

\section{Data analysis}

Two neurologists independently evaluated the ease of application of the prognostic model in clinical practice in terms of the time needed for calculation of an outcome probability for each prognostic model. The assessment was based on the time needed to collect and review all data needed for the model and the availability of these data on the ward on the day of admission. A prognostic model was classified as "easy to score" if calculation would cost no more than 10 minutes on the ward. In case of disagreement, consensus was reached in a meeting chaired by an epidemiologist.

To evaluate whether major treatment decisions could be made based on the predictions of a model, we calculated the highest possible predicted probability that could be generated with each model for the combination of predictors that provided the highest probability of death or poor outcome. We considered a probability of death or poor outcome of $95 \%$ or more high enough as basis for major treatment decisions. In addition, we used a value of $90 \%$ to assess the influence of the cut-off. If a model could not generate predicted probabilities of $90 \%$ or higher, we did not consider the predictions of this model suitable for major treatment or care decisions. For the other prediction models, we calculated for each patient in the validation series the predicted probability for that certain model. Then, for each model, the proportion of patients with a $95 \%$ or higher probability of death or poor outcome was calculated. In addition, we also calculated this proportion for a $90 \%$ cut-off. If we had no data on one categorical predictor, to limit missings, we used its maximum value and estimated the maximum proportion of patients with a probability of $90 \%$ or $95 \%$ or higher. In addition, we also used the minimum value to assess the influence of our assumption. Finally, the 30 day case fatality (proportion of patients who died within 30 days) in patients from our series with a probability of $90 \%$ or $95 \%$ or higher was calculated to assess the accuracy of the prediction.

\section{RESULTS}

We included 18 prognostic models in our analysis (fig 1). ${ }^{6}{ }^{13-29}$ Often reported predictors were haematoma size, presence of intraventricular extension, and a poor clinical condition on admission (table 1). 
Table 1 Study characteristics of the included studies on predictors of death or poor outcome after intracranial haemorrhage

\begin{tabular}{|c|c|c|c|c|c|}
\hline Study & Year & Patients & Beta & Predictors* & Outcome \\
\hline $\begin{array}{l}\text { Portenoy } \\
\text { et } a l^{13}\end{array}$ & 1987 & 112 & $\begin{array}{l}+0.39 \\
-0.07 \\
-1.65 \\
-1.72\end{array}$ & $\begin{array}{l}\text { GCS } \\
\text { Relative haemorrhage volume (proportion) } \\
0.04-0.19 \text { (14); } 0.20-0.28(24) ; 0.29-0.44(36) ; 0.45-0.81 \text { (60) } \\
\text { Intraventricular spread } \\
\text { Intercept }\end{array}$ & $\begin{array}{l}\text { Good outcome } \\
\text { Complement: poor outcome }\end{array}$ \\
\hline $\begin{array}{l}\text { Tuhrim } \\
\text { et } a l^{14}\end{array}$ & 1988 & 94 & $\begin{array}{l}+2.90 \\
+0.91 \\
+0.88 \\
-4.06\end{array}$ & $\begin{array}{l}\text { GCS: } 3-8(1) \vee 9-15(0) \\
\text { Pulse pressure }(\mathrm{mm} \mathrm{Hg}): \leqslant 40(0) ; 41-65(1) ;>65(2) \\
\text { ICH size (lobes): }<1 / 2(0) ; 1 / 2-1(1) ;>1(2) \\
\text { Intercept }\end{array}$ & Death ( 30 days) \\
\hline $\begin{array}{l}\text { Franke } \\
\text { et } a l^{15}\end{array}$ & 1992 & 157 & $\begin{array}{l}+2.64 \\
+1.70 \\
+1.36 \\
+1.28 \\
-5.02\end{array}$ & $\begin{array}{l}\text { Pineal gland displacement }(\mathrm{mm}): \geqslant 3(1) v<3(0) \\
\text { Blood glucose level }(\mathrm{mmol} / \mathrm{l}): \geqslant 8(1) v<8(0) \\
\text { GCS: } \leqslant 8(1) v>8(0) \\
\text { Haemorrhage volume (best fitt) }\left(\mathrm{cm}^{3}\right): \geqslant 40(1) v<40(0) \\
\text { Intercept† }\end{array}$ & Death ( 2 days) \\
\hline $\begin{array}{l}\text { Lisk } \\
\text { et }^{16}{ }^{16}\end{array}$ & 1994 & 75 & $\begin{array}{l}+0.05 \\
-0.34 \\
+0.01 \\
-1.41 \\
+1.55\end{array}$ & $\begin{array}{l}\text { Age } \\
\text { GCS } \\
\text { Haemorrhage volume }(A B C / 2 \uparrow)\left(\mathrm{cm}^{3}\right) \\
\text { Sex } \\
\text { Intercept }\end{array}$ & Poor outcome (discharge/30 days) \\
\hline 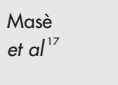 & 1995 & 138 & $\begin{array}{l}-1.75 \\
-0.02\end{array}$ & $\begin{array}{l}\text { Intraventricular spread } \\
\text { Haemorrhage volume }\left(4 / 3^{*} \pi^{*} a b c \xi\right)\left(\mathrm{cm}^{3}\right):<8.3(2.7) ; 8.3-20.4(11.4) ; 20.5-47.7(28.8) \text {; } \\
>47.7(76.8)\end{array}$ & Survival ( 30 days) \\
\hline & & & $\begin{array}{l}+0.25 \\
-0.52\end{array}$ & $\begin{array}{l}\text { GCS } \\
\text { Intercept }\end{array}$ & Complement: death ( 30 days) \\
\hline $\begin{array}{l}\text { Qureshi } \\
\text { et } \text { al }^{18}\end{array}$ & 1995 & 182 & $\begin{array}{l}+1.90 \\
+1.44 \\
+1.17 \\
-2.05\end{array}$ & $\begin{array}{l}\text { Haemorrhage volume (computerised image analysis) }\left(\mathrm{cm}^{3}\right): \geqslant 30(1) v .<30(0) \\
\text { Ventricular extension } \\
\text { GCS: } \leqslant 12(1) v>12(0) \\
\text { Intercept† }\end{array}$ & Death ( 2 days) \\
\hline $\begin{array}{l}\text { Fogelholm } \\
\text { et al }\end{array}$ & 1997 & 282 & $\begin{array}{l}+1.62 \\
+0.03 \\
+1.51 \\
+0.13 \\
+0.18 \\
+0.93 \\
-9.01\end{array}$ & $\begin{array}{l}\text { Level of consciousness: unconscious/comatose (2), somnolent/disoriented (1), alert (0) } \\
\text { Mean arterial pressure }(\mathrm{mm} \mathrm{Hg}) \\
\text { Subarachnoid spread } \\
\text { Shift of midline structures }(\mathrm{mm}) \\
\text { Admission blood glucose }(\mathrm{mmol} / \mathrm{l}) \\
\text { Vomiting on admission } \\
\text { Intercept }\end{array}$ & Death (28 days) \\
\hline $\begin{array}{l}\text { Razzaq and } \\
\text { Hussain }^{20}\end{array}$ & 1998 & 146 & $\begin{array}{r}+0.88 \\
+2.40 \\
+1.10 \\
+0.26 \\
+0.92 \\
+1.06 \\
-21.9\end{array}$ & $\begin{array}{l}\text { GCS: 9-11 (1) } v \geqslant 12(0) \\
\text { GCS: } \leqslant 8(1) v \geqslant 12(0) \\
\text { History of hypertension } \\
\text { Pulse pressure (mm Hg) } \\
\text { Diameter haemorrhage: }>3 \mathrm{~cm}(1) v \leqslant 3 \mathrm{~cm} \mathrm{(0)} \\
\text { Intraventricular spread } \\
\text { Intercept† }\end{array}$ & $\begin{array}{l}\text { Survival ( } 30 \text { days) } \\
\text { Complement: death ( } 30 \text { days) }\end{array}$ \\
\hline $\begin{array}{l}\text { Tuhrim } \\
\text { et } a l^{21}\end{array}$ & 1999 & 129 & $\begin{array}{l}+2.79 \\
+0.02 \\
+0.58 \\
-0.96 \\
+0.10 \\
-3.31\end{array}$ & $\begin{array}{l}\text { GCS: } \leqslant 8(1) v .>8(0) \\
\text { Intraparenchymal haemorrhage volume (computerised image analysis) }\left(\mathrm{cm}^{3}\right) \\
\text { Pulse pressure: }>85 \mathrm{~mm} \mathrm{Hg}(1) v \leqslant 85(0) \\
\text { Hydrocephalus } \\
\text { Intraventricular haemorrhage volume (computerised image analysis) }\left(\mathrm{cm}^{3}\right) \\
\text { Intercept }\end{array}$ & Death (30 days) \\
\hline $\begin{array}{l}\text { Schwarz } \\
\text { et } a l^{22}\end{array}$ & 2000 & 251 & $\begin{array}{l}+1.47 \\
-0.21 \\
+2.18 \\
+1.77 \\
+1.00 \\
+2.73 \\
+0.07 \\
+2.05 \\
-5.37\end{array}$ & $\begin{array}{l}\text { Intraventricular haemorrhage } \\
\text { GCS: } 8-13(1) v 14 \text { or } 15(0) \\
\text { GCS: } 3-7 \text { (1) } v 14 \text { or } 15(0) \\
\text { Coagulation disorder } \\
\text { Age: } 55-75(1) v<55(0) \\
\text { Age: }>75(1) v<55(0) \\
\text { Haemorrhage volume }\left(4 / 3^{*} \pi^{*} \text { abc }\right)\left(\mathrm{cm}^{3}\right): 25-60(1) v<25(0) \\
\text { Haemorrhage volume }\left(4 / 3^{*} \pi^{*} a b c \xi\right)\left(\mathrm{cm}^{3}\right):>60(1) v<25(0) \\
\text { Intercept† }\end{array}$ & Poor outcome (in hospital) \\
\hline $\begin{array}{l}\text { el Chami } \\
\text { et }\left.a\right|^{23}\end{array}$ & 2000 & 180 & $\begin{array}{l}+1.76 \\
+1.72 \\
+1.26 \\
+1.59 \\
+0.08 \\
+0.29 \\
-1.61\end{array}$ & $\begin{array}{l}\text { Consciousness disorders (our definition GCS } \leqslant 8 v>8 \text { ) } \\
\text { Intraventricular haemorrhage } \\
\text { Haemorrhage volume }(A B C / 2)\left(\mathrm{cm}^{3}\right):>11(1) v \leqslant 11(0) \\
\text { Male } \geqslant 70 \text { years }(1) v \text { male }<70 \text { years }(0) \\
\text { Female }<70 \text { years }(1) v \text { male }<70 \text { years }(0) \\
\text { Female } \geqslant 70 \text { years (1) } v \text { male }<70 \text { years (0) } \\
\text { Intercept }\end{array}$ & Death (30 days) \\
\hline $\begin{array}{l}\text { Berwaerts } \\
\text { et } a l^{24}\end{array}$ & 2000 & 42 & $\begin{array}{l}+0.10 \\
-2.78 \\
-3.87\end{array}$ & $\begin{array}{l}\text { Maximal diameter of } \mathrm{ICH} \text { on } \mathrm{CT}(\mathrm{mm}) \\
\text { Signs of cerebral ischaemia on CT } \\
\text { Intercept }\end{array}$ & Death (in hospital) \\
\hline $\begin{array}{l}\text { Phan } \\
\text { et } a l^{25}\end{array}$ & 2000 & 99 & $\begin{array}{l}+2.82 \\
+1.70 \\
-2.37\end{array}$ & $\begin{array}{l}\text { GCS: } \leqslant 8(1) v>8(0) \\
\text { Hydrocephalus based on visual analysis } \\
\text { Interceptt }\end{array}$ & Death (30 days) \\
\hline $\begin{array}{l}\text { Hemphill } \\
\text { et } a l^{6}\end{array}$ & 2001 & 152 & $\begin{array}{l}-0.37 \\
+2.29 \\
+1.44 \\
+1.09 \\
+0.27 \\
+0.52\end{array}$ & $\begin{array}{l}\text { GCS: } 13-15(2) ; 5-12(1) ; 3-4(0) \\
\text { Age: } \geqslant 80(1) v<80(0) \\
\text { ICH location: infratentorial }(1) \text { v supratentorial }(0) \\
\text { Intraventricular haemorrhage } \\
\text { Haemorrhage volume }(A B C / 29 \text {, divided by } 10)\left(\mathrm{cm}^{3}\right) \\
\text { Interceptt }\end{array}$ & Death (30 days) \\
\hline $\begin{array}{l}\text { Passero } \\
\text { et }\left.a\right|^{26}\end{array}$ & 2002 & 26 & $\begin{array}{l}+1.54 \\
+1.60 \\
-1.53\end{array}$ & $\begin{array}{l}\text { GCS: } \leqslant 8(1) v>8(0) \\
\text { Early hydrocephalus } \\
\text { Interceptt }\end{array}$ & Death (in hospital) \\
\hline
\end{tabular}


Table 1 (continued) Study characteristics of the included studies on predictors of death or poor outcome after intracranial haemorrhage

\begin{tabular}{|c|c|c|c|c|c|}
\hline Study & Year & Patients & Beta & Predictors* & Outcome \\
\hline $\begin{array}{l}\text { Hallevy } \\
\text { et al }{ }^{27}\end{array}$ & 2002 & 184 & $\begin{array}{l}+0.56 \\
+1.01 \\
+0.45 \\
+0.51 \\
+0.29 \\
+0.56 \\
-1.31\end{array}$ & $\begin{array}{l}\text { Age: }>60 \text { (1) } v \leqslant 60(0) \\
\text { Hemiparesis severe: } 2 / 5-0 / 5(1) \text { v } 0 / 5-3 / 5(0) \\
\text { Consciousness: drowsy/comatose (1) v alert (0) } \\
\text { Midline shift } \\
\text { Haemorrhage volume (radiologist impression): large (1) v small/medium (0) } \\
\text { Intraventricular spread } \\
\text { Intercept† }\end{array}$ & Poor outcome (discharge) \\
\hline $\begin{array}{l}\text { Nilsson } \\
\text { et al }{ }^{28}\end{array}$ & 2002 & 341 & $\begin{array}{l}+1.65 \\
+3.74 \\
+0.26 \\
+1.28 \\
+0.88 \\
-2.19\end{array}$ & $\begin{array}{l}\text { GCS: 8-13 (1) v 14-15 (0) } \\
\text { GCS: 3-7 (1) v 14-15(0) } \\
\text { Haemorrhage volume }(\mathrm{ABC} / 2 \uparrow)\left(\mathrm{cm}^{3}\right): 30-60(1) v<30(0) \\
\text { Haemorrhage volume }(\mathrm{ABC} / 2 \uparrow)\left(\mathrm{cm}^{3}\right):>60(1) v<30(0) \\
\text { Heart disease } \\
\text { Intercept† }\end{array}$ & Death (30 days) \\
\hline $\begin{array}{l}\text { Szczudlik } \\
\text { et }\left.a\right|^{29}\end{array}$ & 2002 & 152 & $\begin{array}{l}-0.08 \\
+0.03 \\
+0.86 \\
+0.71 \\
+0.39 \\
-0.04 \\
-1.29\end{array}$ & $\begin{array}{l}\text { Neurological deficit (Scandinavian Stroke Scale score) } \\
\text { Age } \\
\text { Midline shift on CT } \\
\text { Hyperthermia on day } 1:>37.5^{\circ} \mathrm{C}(1) v<37.5^{\circ} \mathrm{C}(0) \\
\text { Diameter haematoma on } \mathrm{CT}: \geqslant 15 \mathrm{~mm}(1) v<15 \mathrm{~mm}(0) \\
\text { Sex } \\
\text { Intercept† }\end{array}$ & Death (30 days) \\
\hline $\begin{array}{l}\text { Unless cod } \\
\text { *Value in } 1 \\
\text { †Intercept } \\
\text { †Franke et } \\
\text { CT, compu }\end{array}$ & $\begin{array}{l}\text { otherw } \\
\text { represe } \\
\text { lculated } \\
I^{30} ; \boldsymbol{\uparrow} \text { Ko } \\
\text { d tomoc }\end{array}$ & $\begin{array}{l}\text { tegorical } \\
\text { value wit } \\
\text { absolute } p \\
\text { et } \text { al }^{31} ; \S B \\
\text { GCS, G }\end{array}$ & $\begin{array}{l}\text { ariables } \\
\text { which } t \\
\text { obability } \\
\text { oderick } \\
\text { asgow C }\end{array}$ & $\begin{array}{l}\text { coded as present ( } 1 \text { ) and absent }(0) \text { and sex as male (1) v female (0). } \\
\text { ta has to be multiplied in the regression equation. } \\
\text { ression coefficients and mean values of the variables. } \\
\text { Scale; } I C H \text {, intracerebral haemorrhage. }\end{array}$ & \\
\hline
\end{tabular}

Fourteen prognostic models were classified as easy to score (table 2). The highest possible predicted probability of death or poor outcome for the combination of predictors that provided the highest probability of death or poor outcome ranged from $80 \%$ to $100 \%$ (median $99 \%$ ) (see table 2 ).

The characteristics of the patients included in the validation series are shown in table 3. Thirty day mortality was $40 \%$. Patients who died within 30 days were slightly older, had a higher blood pressure, a larger ICH volume, a lower score on the GCS on admission, and more often ventricular extension of the haemorrhage (table 3). These data are consistent with the predictors suggested by the studies shown in table 1 . Therefore, we assumed that our series of patients with ICH is a good representation of patients who are admitted to a hospital with an ICH.

In the validation series, the proportion of patients with a $\geqslant 95 \%$ probability of poor outcome according to eight different models ranged from $0 \%$ to $43 \%$ (median 23\%). Seven models could identify a subset of patients with $a \geqslant 95 \%$ probability of death or poor outcome. In these subsets, the 30 day case fatality for the seven prognostic models ranged from $75 \%$ to $100 \%$ (median $93 \%$; see table 2 ) Ten prognostic models could identify a subset of patients with a $\geqslant 90 \%$ probability of death or poor outcome in the validation series.

Table 2 Assessment of the clinical applicability, relevance, and discriminatory power of the models

\begin{tabular}{|c|c|c|c|c|c|c|}
\hline \multirow[b]{2}{*}{ Study } & \multirow[b]{2}{*}{ Year } & \multirow[b]{2}{*}{$\begin{array}{l}\text { Easy to } \\
\text { score }\end{array}$} & \multirow{2}{*}{$\begin{array}{l}\text { Highest predicted probability of } \\
\text { death or poor outcome generated } \\
\text { by the model for patients with a } \\
\text { combination of predictors } \\
\text { providing the worst prognosis }\end{array}$} & \multicolumn{2}{|l|}{ Relevance of the prognostic model* } & \multirow[b]{2}{*}{$\begin{array}{l}\text { Area under the ROC } \\
\text { curve }(95 \% \mathrm{CI})\end{array}$} \\
\hline & & & & $\begin{array}{l}\text { Patients with } \geqslant 95 \% \text { probability of } \\
\text { death or poor outcome } \\
\text { (n }(\%) \text { ) }\end{array}$ & $\begin{array}{l}30 \text { day case fatalityt of patients with } \\
\geqslant 95 \% \text { probability of death or poor } \\
\text { outcome }(95 \% \text { CI) }\end{array}$ & \\
\hline Portenoy et al ${ }^{13}$ & 1987 & + & $100 \%$ & $52(43 \%)^{*}$ & $75 \%(61 \%$ to $86 \%)$ & $0.87(0.79$ to 0.95$)$ \\
\hline Tuhrim et al ${ }^{14}$ & 1988 & + & $92 \%$ & - & - & $0.87(0.79$ to 0.95$)$ \\
\hline Franke et $\left.a\right|^{15}$ & 1992 & + & $88 \%$ & - & - & $0.87(0.78 \text { to } 0.95)^{*}$ \\
\hline Lisk et $a l^{16}$ & 1994 & + & $100 \%$ & $26(21 \%)$ & $96 \%(80 \%$ to $100 \%)$ & $0.86(0.78$ to 0.94$)$ \\
\hline Masè et al ${ }^{17}$ & 1995 & + & $97 \%$ & $3(3 \%)$ & $100 \%(29 \%$ to $100 \%)$ & $0.89(0.83$ to 0.96$)$ \\
\hline Qureshi ef $a l^{18}$ & 1995 & - & $92 \%$ & - & - & $0.87(0.80$ to 0.94$)$ \\
\hline Fogelholm et al ${ }^{19}$ & 1997 & - & $100 \%$ & $\ddagger$ & $\ddagger$ & $\ddagger$ \\
\hline $\begin{array}{l}\text { Razzaq and } \\
\text { Hussain }\end{array}$ & 1998 & + & $100 \%$ & $\ddagger$ & $\ddagger$ & $\ddagger$ \\
\hline Tuhrim et al ${ }^{21}$ & 1999 & - & $100 \%$ & $3(2 \%)$ & $100 \%(29 \%$ to $100 \%)$ & $0.90(0.83$ to 0.96$)$ \\
\hline Schwarz et $a l^{22}$ & 2000 & + & $99 \%$ & $0(0 \%)^{*}$ & - & $0.81(0.72 \text { to } 0.91)^{*}$ \\
\hline el Chami et al ${ }^{23}$ & 2000 & + & $99 \%$ & 40 (33\%) & $85 \%$ (70\% to $94 \%)$ & $0.86(0.78$ to 0.95$)$ \\
\hline Berwaerts ef $a l^{24}$ & 2000 & + & $100 \%$ & $\ddagger$ & $\ddagger$ & $\ddagger$ \\
\hline Phan et $\left.a\right|^{25}$ & 2000 & + & $80 \%$ & - & - & $\ddagger$ \\
\hline Hemphill et $a l^{6}$ & 2001 & + & $100 \%$ & $42(34 \%)$ & $79 \%$ (63\% to $90 \%)$ & 0.86 (0.79 to 0.93 ) \\
\hline Passero et $a^{26}$ & 2002 & + & $83 \%$ & - & - & $\ddagger$ \\
\hline Hallevy ef $a l^{27}$ & 2002 & - & $92 \%$ & - & - & $\ddagger$ \\
\hline Nilsson et $a^{28}$ & 2002 & + & $98 \%$ & $29(24 \%)$ & $93 \%$ (77\% to $99 \%)$ & 0.89 (0.83 to 0.95$)$ \\
\hline Szczudlik et $a l^{29}$ & 2002 & + & $98 \%$ & $\ddagger$ & $\ddagger$ & $\ddagger$ \\
\hline
\end{tabular}

An area under the curve of 1 corresponds with a perfect prediction and an area under the curve of 0.5 with no discriminatory power at all.

Franke et $a l^{15}$ and Qureshi et $a l^{18}$ developed models to predict outcome at two days after admission.

*Results from validation series $(n=122)$. Probabilitydeath/poor outcome $\geqslant 95 \%$.

tInformation on one predictor was missing, the highest possible value was imputed for all patients and the proportion was estimated-for example, for Portenoy et al in our series we had no information on relative haemorrhage volume, therefore we imputed the highest value possible and estimated the maximum percentage. These proportions are an overestimation of the true proportions (minimum proportion (30 day case fatality): Portenoy $4 \%$ (100\%; $95 \%$ Cl $48 \%$ to $100 \%$ ); Nilsson $0 \%(-)$.

‡ It was not possible to calculate this percentage, because one or more of the predictors were not measured in our series or because predictors were ill defined. 
Table 3 Patient characteristics of the validation series of patients with intracerebral haemorrhage

\begin{tabular}{|c|c|c|c|}
\hline Patient characteristics* & Dead at 30 days ( $n=49 ; 40 \%$ ) & Alive at 30 days ( $n=73 ; 60 \%$ ) & Total $(n=122)(100 \%)$ \\
\hline Sex (male) & $25(51 \%)$ & $41(56 \%)$ & $66(54 \%)$ \\
\hline Age (years) & $66(14)$ & 64 (17) & $65(16)$ \\
\hline Systolic blood pressure $(\mathrm{mm} \mathrm{Hg})$ & $194(41)(n=34 ;$ missing = 15) & $170(36)(n=68 ;$ missing $=5)$ & $178(39)(n=102 ;$ missing $=20)$ \\
\hline Diastolic blood pressure $(\mathrm{mm} \mathrm{Hg})$ & $108(25)(n=34 ;$ missing $=15)$ & $100(22)(n=68 ;$ missing $=5)$ & $103(23)(n=102 ;$ missing $=20)$ \\
\hline Glasgow Coma Scale† & $5(3-15)$ & $15(5-15)$ & $12(3-15)$ \\
\hline Intracerebral haemorrhage volume $\left(\mathrm{cm}^{3}\right)$ & $92(63)$ & $33(30)$ & $56(54)$ \\
\hline Intraventricular spread (yes) & 39 (80\%) & $26(36 \%)$ & 65 (53\%) \\
\hline Volume ventricular haemorrhage $\left(\mathrm{cm}^{3}\right)$ & $16(20)$ & $3(6)$ & $8.6(15)$ \\
\hline Septum pellucidum shift (mm) & $1.3(0.5)$ & $0.44(0.40)$ & $0.78(0.62)$ \\
\hline Hydrocephalus (present) & $20(41 \%)$ & $32(44 \%)$ & $52(43 \%)$ \\
\hline
\end{tabular}

*Continuous variables: mean (SD); categorical variables: $\mathrm{n}(\%)$.

†Median (range)

The proportion of patients with such a probability ranged from $5 \%$ to $48 \%$ (median $30 \%$ ). In these subsets, the 30 day case fatality ranged from $67 \%$ to $100 \%$ (median $89 \%$, data not shown).

We computed an ROC curve for each model to evaluate the prediction of outcome over the whole range of outcome probabilities relevant to inform patients or their relatives about their prognosis. The AUC ranged from 0.81 to 0.90 (see table 2).

\section{DISCUSSION}

In the present study, most prediction models for outcome after ICH appeared easy to apply and most could generate a high probability of death or poor outcome in patients with the combination of predictors providing the highest probability. However, only a small proportion of patients admitted to a hospital with ICH have such a high probability of death or poor outcome. In addition, 30 day case fatality was not always correctly predicted in the validation series. Because these models leave too much uncertainty for most patients and sometimes give an incorrect prediction, their relevance in deciding whether or not to start intensive treatment is limited. We estimated the discriminatory power of all the models with ROC curves. Every model had a reasonable AUC, with a value closer to one than to zero. Therefore, the estimations of prognosis obtained from these models can be used to give an estimate to patients and relatives of the chance of survival of the patient.

We focused our evaluation on the use of prognostic models for application soon after the first clinical and radiological assessment because at that stage prediction of outcome makes an important contribution to major treatment decisions. If either surgery or change in a predictor over time is included as a predictor of outcome, a prediction rule cannot be used for such early decision making. We had to limit our evaluation to studies in which the intercept was reported or from which we could calculate the intercept. Because of this criterion three studies were excluded-none of these reported a model with predictors that were strongly associated with death or poor outcome.

We used a series of 122 patients to assess the clinical relevance of the models. Our series was approximately the same size as the series in which the prediction models had been developed. The limitations of our series were first, the data were collected retrospectively, and second, of more than 300 patients who were eventually admitted to our hospital only 122 were eligible for this validation series. Nevertheless, our validation series had approximately the same patient characteristics, and furthermore, the factors associated with death within 30 days were similar to the factors identified in the studies in which the models were developed. Therefore, our validation series seems to be a representative sample of patients admitted to a hospital with ICH.
With regard to the analyses to assess clinical relevance we made the assumption that a probability of poor outcome of $95 \%$ or higher was high enough to base treatment decisions upon. This cut-off value was based on the assumption that intensive treatment might not be started in some patients with less than a $5 \%$ chance of survival. We used a value of $90 \%$ to assess the influence of this cut-off, and the results did not change significantly with it. It is known that prediction rules predict less well in their extremes. However, for treatment decisions pertaining to life and death one has to be certain about the prediction of the outcome. One could also plead for a higher cut-off value, however, in that case even fewer patients would have had such a high predicted probability and clinical relevance would be limited.

Several comments can be made on the methodological quality of studies on clinical prediction rules. ${ }^{33}{ }^{34}$ The first pertains to outcome measures. In most studies the only outcome measure was mortality ${ }^{6} 1415$ 17-21 23-26 28 29; only four studies included poor outcome based on the GCS or the modified Rankin scale. ${ }^{13} 162227$ From the patients' perspective poor outcome may be a better outcome measure than death. The second point to consider addresses the predictors. The definitions of the predictors were not clearly described in a number of studies, thus, other investigators cannot apply these rules. One study did not define how to classify level of consciousness. ${ }^{19}$ For use of a prediction rule in clinical practice clear predictor definitions are essential. In 11 studies, the data were obtained retrospectively, with the inherent risk of missing or biased data on predictors. ${ }^{6}{ }^{13} 16-2022242527$ Lastly, only two models were externally validated ${ }^{2635}$ : one used patients from the same stroke registry ${ }^{14}$ and the other was validated by a research group different from the group that developed the model. ${ }^{6}$

In the development of a prediction model, about ten outcome events are needed for the inclusion of one predictor. ${ }^{36}$ Because approximately half the patients admitted to a hospital with ICH die, it should be possible to develop a valid model. However, for a model to be used in treatment decisions, it has to predict outcome extremely well to avoid errors with serious consequences. Key factors that should be included are the clinical condition on admission and the extent of the haemorrhage. Since these factors do not predict well enough for the majority of patients, other factors should be added. Factors that seem worth studying are the course of the clinical condition within the first hours after admission and the size of the haematoma on a repeated scan a few hours after admission (because haematomas enlarge within the first hours after the haemorrhage).

We conclude that the current prediction rules for predicting high probabilities of poor outcome after ICH are not sufficiently accurate, or can predict for only a small proportion of patients, to base decisions about commencement of intensive treatment upon. A useful model in clinical 
practice should give a very precise prediction for a large group of patients. However, the current models do have reasonable discriminatory power and can therefore be used to estimate the chances of survival of individual patients.

\section{ACKNOWLEDGEMENTS}

We thank Dr S P Claus for permission to use the data he collected of 122 patients who were admitted with an ICH to the University Medical Centre Utrecht. We thank Prof Dr D E Grobbee for constructive comments on an earlier version of this manuscript.

\section{Authors' affiliations}

M J Ariesen, A Algra, Julius Centre for Health Sciences and Primary Care, University Medical Centre Utrecht, the Netherlands A Algra, H B van der Worp, G J E Rinkel, Department of Neurology, Rudolf Magnus Institute of Neuroscience, University Medical Centre Utrecht, the Netherlands

This research was supported by the Health Research and Development Counsel of the Netherlands (ZonMw, project number 904-61-190). This study was in part supported by an established clinical investigator grant from the Netherlands Heart Foundation to Prof Dr G J E Rinkel Igrant D98.014)

Competing interests: none declared

\section{REFERENCES}

1 Keir SL, Wardlaw JM, Warlow CP. Stroke epidemiology studies have underestimated the frequency of intracerebral haemorrhage. A systematic review of imaging in epidemiological studies. J Neurol 2002;249:1226-31.

2 Bamford J, Sandercock P, Dennis M, et al. A prospective study of acute cerebrovascular disease in the community: the Oxfordshire Community Stroke Project-1981-86. 2. Incidence, case fatality rates and overall outcome at one year of cerebral infarction, primary intracerebral and subarachnoid haemorrhage. J Neurol Neurosurg Psychiatry 1990;53:16-22.

3 Giroud M, Gras P, Chadan N, et al. Cerebral haemorrhage in a French prospective population study. J'Neurol Neurosurg Psychiatry 1991;54:595-8.

4 Thrift AG, Dewey HM, Macdonell RA, et al. Incidence of the major stroke subtypes: initial findings from the North East Melbourne stroke incidence study (NEMESIS). Stroke 2001;32:1732-8.

5 Hanel RA, Xavier AR, Mohammad Y, et al. Outcome following intracerebral hemorrhage and subarachnoid hemorrhage. Neurol Res 2002;24(suppl 1):S58-S62.

6 Hemphill JC III, Bonovich DC, Besmertis L, et al. The ICH score: a simple, reliable grading scale for intracerebral hemorrhage. Stroke 2001;32:891-7.

7 van Swieten JC, Koudstaal PJ, Visser MC, et al. Interobserver agreement for the assessment of handicap in stroke patients. Stroke 1988;19:604-7.

8 Rankin J. Cerebral vascular accidents in patients over the age of 60. II. Prognosis. Scott Med J 1957;2:200-15.

9 Jennett B, Bond M. Assessment of outcome after severe brain damage. Lancet $1975 ; 1: 480-4$

10 Van Gijn J, Hijdra A, Wijdicks EF, et al. Acute hydrocephalus after aneurysmal subarachnoid hemorrhage. J Neurosurg 1985;63:355-62.

11 Swets JA. Measuring the accuracy of diagnostic systems. Science 1988;240:1285-93.

12 Hanley JA, McNeil BJ. The meaning and use of the area under a receiver operating characteristic (ROC) curve. Radiology 1982;143:29-36.
13 Portenoy RK, Lipton RB, Berger AR, et al. Intracerebral haemorrhage: a model for the prediction of outcome. J Neurol Neurosurg Psychiatry 1987;50:976-9.

14 Tuhrim S, Dambrosia JM, Price TR, et al. Prediction of intracerebral hemorrhage survival. Ann Neurol 1988;24:258-63.

15 Franke CL, van Swieten JC, Algra A, et al. Prognostic factors in patients with intracerebral haematoma. J Neurol Neurosurg Psychiatry 1992;55:653-7.

16 Lisk DR, Pasteur W, Rhoades $\mathrm{H}$, et al. Early presentation of hemispheric intracerebral hemorrhage: prediction of outcome and guidelines for treatment allocation. Neurology 1994;44:133-9.

17 Masè G, Zorzon M, Biasutti $E$, et al. Immediate prognosis of primary intracerebral hemorrhage using an easy model for the prediction of survival. Acta Neurol Scand 1995:91:306-9.

18 Qureshi Al, Safdar K, Weil J, et al. Predictors of early deterioration and mortality in black Americans with spontaneous intracerebral hemorrhage. Stroke 1995;26:1764-7.

19 Fogelholm R, Avikainen S, Murros K. Prognostic value and determinants of first-day mean arterial pressure in spontaneous supratentorial intracerebral hemorrhage. Stroke 1997;28:1396-400.

20 Razzaq AA, Hussain R. Determinants of 30-day mortality of spontaneous intracerebral hemorrhage in Pakistan. Surg Neurol 1998;50:336-42.

21 Tuhrim S, Horowitz DR, Sacher M, et al. Volume of ventricular blood is an important determinant of outcome in supratentorial intracerebral hemorrhage. Crit Care Med 1999;27:617-21.

22 Schwarz S, Hafner K, Aschoff A, et al. Incidence and prognostic significance of fever following intracerebral hemorrhage. Neurology 2000;54:354-61.

23 el Chami B, Milan C, Giroud M, et al. Intracerebral hemorrhage survival: French register data. Neurol Res 2000;22:791-6.

24 Berwaerts J, Dijkhuizen RS, Robb OJ, et al. Prediction of functional outcome and in-hospital mortality after admission with oral anticoagulant-related intracerebral hemorrhage. Stroke 2000;31:2558-62.

25 Phan TG, Koh M, Vierkant RA, et al. Hydrocephalus is a determinant of early mortality in putaminal hemorrhage. Stroke 2000;31:2157-62.

26 Passero S, Ulivelli M, Reale F. Primary intraventricular haemorrhage in adults. Acta Neurol Scand 2002;105:115-19.

27 Hallevy C, Ifergane G, Kordysh E, et al. Spontaneous supratentorial intracerebral hemorrhage. Criteria for short-term functional outcome prediction. J Neurol 2002;249:1704-9.

28 Nilsson OG, Lindgren A, Brandt L, et al. Prediction of death in patients with primary intracerebral hemorrhage: a prospective study of a defined population. J Neurosurg 2002;97:531-6.

29 Szczudlik A, Turaj W, Slowik A, et al. Hyperthermia is not an independent predictor of greater mortality in patients with primary intracerebral hemorrhage. Med Sci Monit 2002;8:CR702-CR707.

30 Franke CL, Versteege CW, Van Gijn J. The best fit method. A simple way for measuring the volume of an intracerebral haematoma. Neuroradiology 1988;30:73-5.

31 Kothari RU, Brott T, Broderick JP, et al. The ABCs of measuring intracerebral hemorrhage volumes. Stroke 1996;27:1304-5.

32 Broderick JP, Brott TG, Duldner JE, et al. Volume of intracerebral hemorrhage. A powerful and easy-to-use predictor of 30-day mortality. Stroke 1993;24:987-93.

33 Wasson JH, Sox HC, Neff RK, et al. Clinical prediction rules. Applications and methodological standards. N Engl J Med 1985;313:793-9.

34 Laupacis A, Sekar N, Stiell IG. Clinical prediction rules. A review and suggested modifications of methodological standards. JAMA 1997;277:488-94.

35 Fernandes H, Gregson BA, Siddique MS, et al. Testing the ICH score. Stroke 2002;33:1455-6.

36 Harrell FE Jr, Lee KL, Matchar DB, et al. Regression models for prognostic prediction: advantages, problems, and suggested solutions. Cancer Treat Rep 1985;69:1071-7. 